\title{
Introduction to the Principle of the AKA-Hakata Method and Sacroiliac Joint Dysfunction
}

\author{
Shigehiko Katada
}

\subsection{Definition of AKA-Hakata Method}

The arthrokinematic approach (AKA) is a method to treat abnormalities of the intra-articular movements such as joint play, sliding, rolling, and spinning of the joint surface. The technique of the AKA-Hakata method is developed based on arthrokinematics, considering articular neurology [1].

\subsection{Pain in Joint Dysfunction}

Musculoskeletal pain could originate from either organic disorder or functional disorder. Although functional disorders are quite prevalent, modern medical standards, including imaging equipment and surgical technology, could not detect functional disorders. Joint dysfunction is one of the essential causes of the so-called musculoskeletal pain. The AKA-Hakata method is a manual technique to treat the joint dysfunction caused by abnormalities of intra-articular movement. There may be simultaneous organic disorder and related functional disorder in the joint. The AKA-Hakata method is also useful to treat functional joint disorder under organic pathological conditions, such as deformity of the knee and hip joint due to osteoarthritis confirmed in X-ray imaging.

A joint dysfunction often occurs particularly in the synovial joint having a small range of motion (small movement joint) such as the sacroiliac joint, facet joints, costovertebral joints, sternoclavicular joints, acromioclavicular joints, carpal joints, subtalar joint, intertarsal joints, and so on. These joints work to support the near joint having a large range of motion (large movement joint) such as hip joint, glenohumeral joint, wrist joint, ankle joint, and so on. When these small movement joints work normally, large movement joints could work normally without stress. However, when abnormalities of the intra-articular movement in the small movement joint occur, the large movement joint by these small movement joints does not move normally.

Restricted joint surface movement in an intra-articular lesion could cause functional joint disorder.

\subsection{Sacroiliac Joint Dysfunction}

Sacroiliac joint dysfunction holds a unique position among various joint dysfunctions, and it may be related to other forms of joint dysfunction. As a result, miscellaneous symptoms appear in not only the sacroiliac joint region but also the systemic regions.

Sacroiliac joint dysfunction frequently causes lower back and buttock pain, and it may be the most frequent cause of the so-called non-specific lower back and buttock pain. We should always consider sacroiliac joint dysfunction as an origin of the pain, even when MRI shows lumbar disc herniation or lumbar spinal canal stenosis, because the patient's symptoms may actually originate from joint dysfunction rather than radiculopathy.

\section{Reference}

1. Hakata S, editor. Arthrokinematic approach Hakata method. 2nd ed. Tokyo: Ishiyaku Shuppan; 2007.

S. Katada $(\bowtie)$

Japanese Medical Society of Arthrokinematic Approach,

Katada Orthopaedic Clinic, Kanagawa, Japan

e-mail: katada@aroma.ocn.ne.jp 